Corrigendum

\title{
Corrigendum to "Surgical Management of a Giant Adrenal Pseudocyst: A Case Report and Review of the Literature in the Last Decade"
}

\author{
Daniel Paramythiotis (D), ${ }^{1}$ Petros Bangeas $\mathbb{D}^{1},{ }^{1}$ Anestis Karakatsanis, ${ }^{1}$ Patroklos Goulas, ${ }^{1}$ \\ Irini Nikolaou, ${ }^{2}$ Vasileios Rafailidis $\left(\mathbb{D},{ }^{2}\right.$ Konstantinos Kouskouras, ${ }^{2}$ \\ Vasileios Papadopoulos, ${ }^{1}$ Sofia Lypiridou, ${ }^{3}$ Georgia Karayannopoulou, ${ }^{3}$ \\ and Antonios Michalopoulos ${ }^{1}$ \\ ${ }^{1} 1$ st Propedeutic Surgical Department, AHEPA University Hospital, Aristotle University of Thessaloniki, Thessaloniki, Greece \\ ${ }^{2}$ Department of Radiology, AHEPA University Hospital of Thessaloniki, Thessaloniki, Greece \\ ${ }^{3}$ Department of Pathology, AHEPA University Hospital of Thessaloniki, Thessaloniki, Greece
}

Correspondence should be addressed to Petros Bangeas; pbangeas@gmail.com

Received 8 March 2018; Accepted 12 March 2018; Published 15 May 2018

Copyright (C) 2018 Daniel Paramythiotis et al. This is an open access article distributed under the Creative Commons Attribution License, which permits unrestricted use, distribution, and reproduction in any medium, provided the original work is properly cited.

In the article titled "Surgical Management of a Giant Adrenal Pseudocyst: A Case Report and Review of the Literature in the Last Decade" [1], there was an error in the legend of Figure 1, which should be corrected as follows:

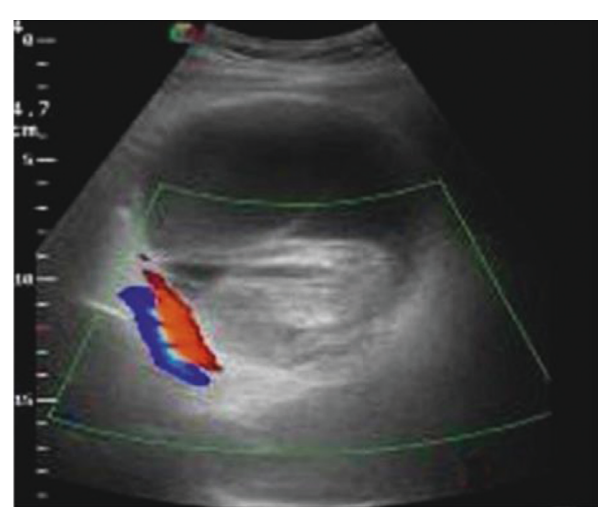

(a)

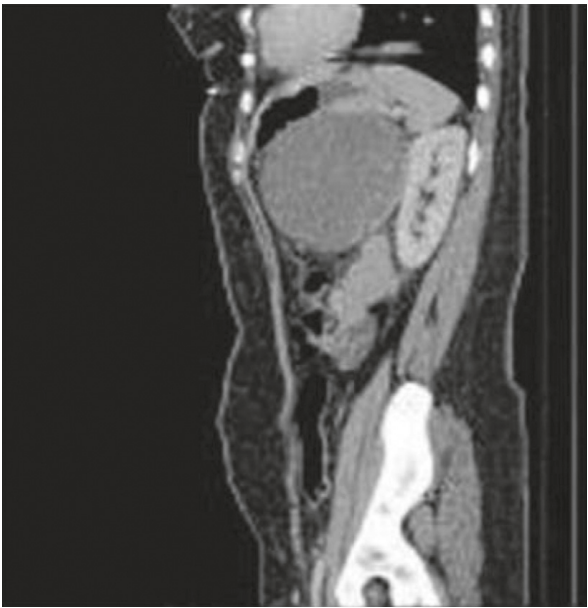

(b)

FIgURE 1: Continued. 


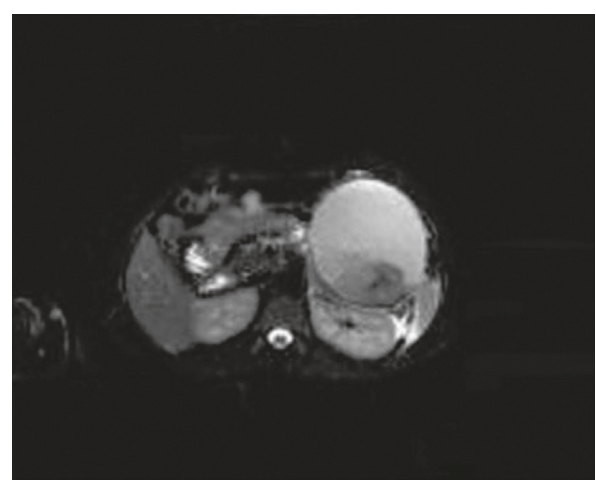

(c)

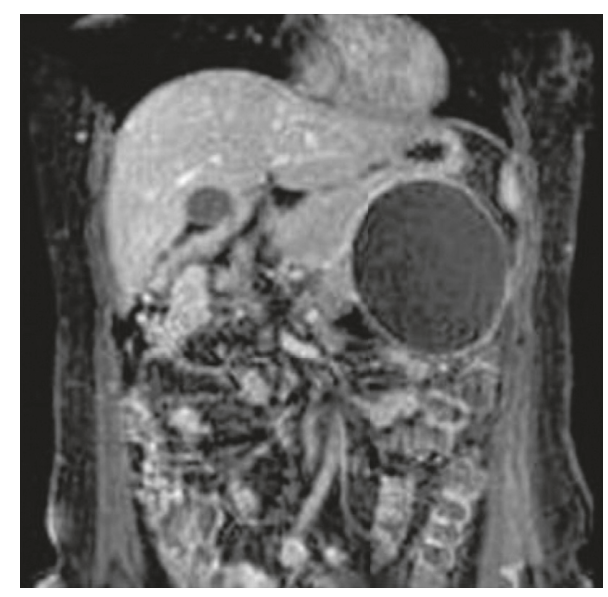

(d)

Figure 1: (a) Colour Doppler ultrasound image showing a cystic lesion containing echogenic debris with no vascularity. (b) Sagittal contrast-enhanced CT image showing the cystic lesion located within the left kidney and the tail of the pancreas. (c) Axial T2-weighted MR image with fat suppression confirming the cystic nature of the lesion. Note: it is made of dependent low-signal intensity debris, in keeping with hemorrhagic content. (d) Coronal contrast-enhanced T1-weighted MR image showing only mild capsular enhancement but no enhancement within the cyst.

\section{References}

[1] D. Paramythiotis, P. Bangeas, A. Karakatsanis et al., "Surgical management of a giant adrenal pseudocyst: a case report and review of the literature in the last decade," Case Reports in Surgery, vol. 2018, Article ID 8473231, 6 pages, 2018. 


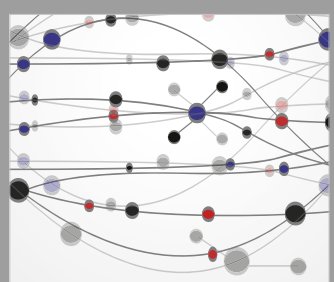

The Scientific World Journal
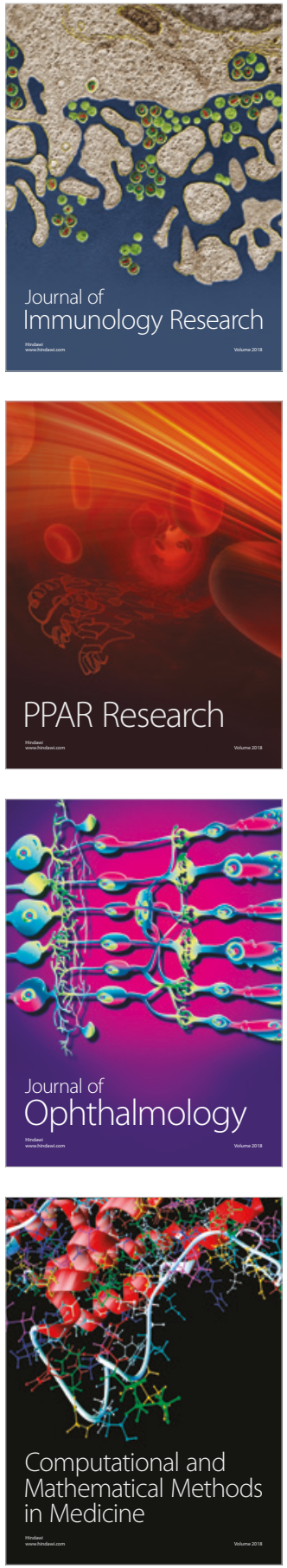

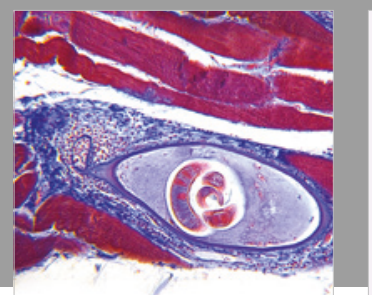

Gastroenterology Research and Practice

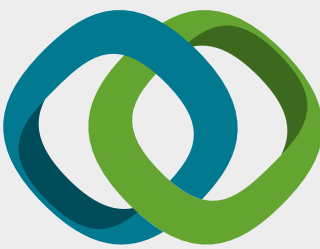

\section{Hindawi}

Submit your manuscripts at

www.hindawi.com
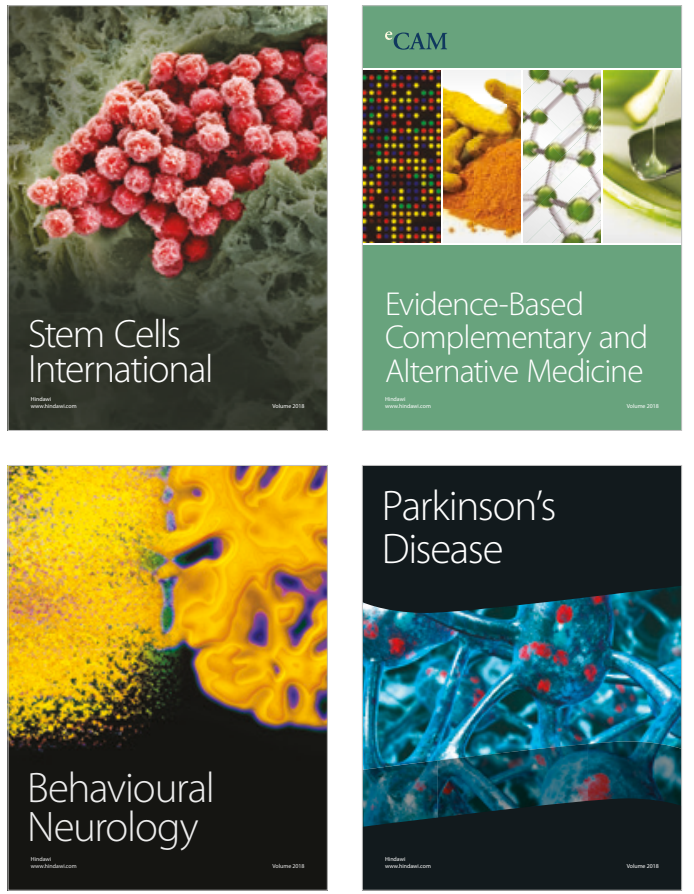

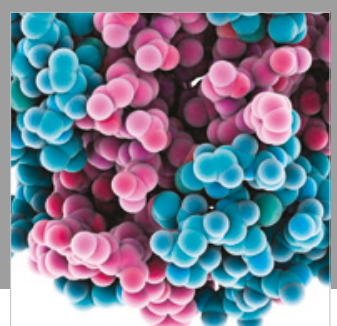

ournal of

Diabetes Research

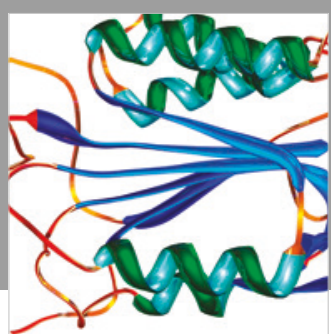

Disease Markers
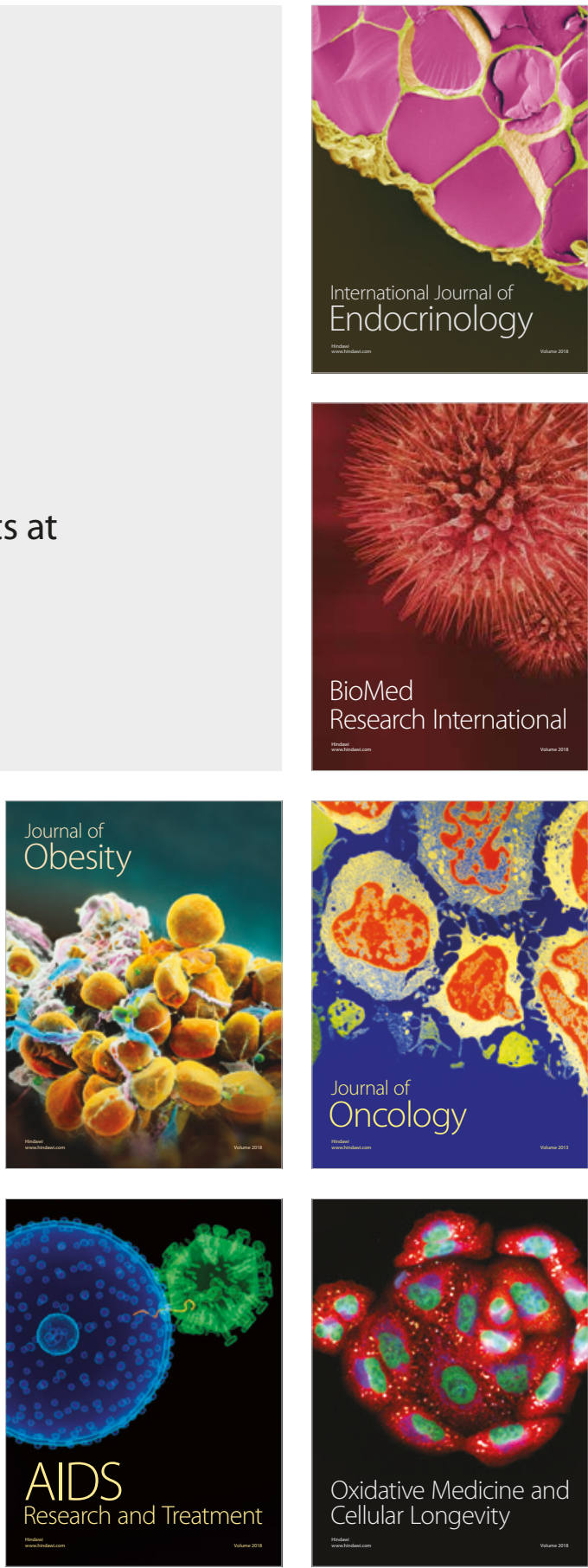